\title{
The design and thermodynamic analysis of electricity-Compressed Natural Gas multi-compression process using natural gas pressure energy
}

\author{
Hui Zhang ${ }^{1, a}$, Xiaxi Li ${ }^{2, b}$, Linlin Xing ${ }^{3, c}$, Qingyu Wang ${ }^{4, d}$ \\ ${ }^{1}$ Full address of first author, including country \\ ${ }^{1}$ No.33, Zhanghua Road, Haidian District, Beijing, China \\ ${ }^{2}$ No.22, South Street in Xizhimen, Haidian District, Beijing, China \\ ${ }^{3}$ No.33, Zhanghua Road, Haidian District, Beijing, China \\ ${ }^{4}$ No.22, South Street in Xizhimen, Haidian District, Beijing, China \\ azhanghui707shui@126.com, b1851322903@qq.com, c253059939@qq.com, \\ d471204473@qq.com
}

\begin{abstract}
Keywords: Natural gas; pressure energy; electricity-CNG; exergy analysis; multi-compression.
Abstract. A novel electricity- Compressed Natural Gas (CNG) multi-compression process using natural gas pressure energy is proposed. Compared to electricity-CNG single-compression process using natural gas pressure energy with the same CNG product, the multi-compression process produces 92 more $\mathrm{kW}$ power. In this paper, exergy analysis and energy utilization diagram method (EUD) are used to assess the new process and identify the key operation units with large exergy loss. The results show that exergy efficiency of the multi-compression process is $57.9 \%$. Compared to the single-compression process, exergy efficiency of the new process is improved by $50.4 \%$. The proposed process has been applied and implemented in a conceptual design scheme of the pressure energy utilization for a 37.5 million $\mathrm{Nm}^{3} /$ year CNG-compression station in some northern Chinese city.
\end{abstract}

\section{Introduction}

With the extreme climate of fog increasingly obvious, the development of new energy and adjustment of energy structure are particularly important. As a kind of clean and high-efficiency energy, natural gas is more and more favored by different countries and regions. Natural gas is usually delivered to each big pressure regulating stations in the form of high-pressure pipeline, and the delivering pressure of pipeline is usually $12 \mathrm{MPa}$ in some European countries, and $10 \mathrm{MPa}$ in China [1]. Natural gas is regulated to medium pressure or low pressure using pressure regulator step by step, then to the downstream users. The process above can release a lot of pressure energy, so it is vital to effectively recycle this part of pressure energy $[2,3]$.

At present, the pressure energy of natural gas pipeline network is mainly used in power generation [4,5], ice-making [6], light hydrocarbon separation [7], liquefaction process [8], refrigerator, air-conditioner and air separation etc. [9,10,11]. Yongqiang Xiong [12] developed a refrigeration process for tire rubber comminuting with pressure energy recovered from natural gas pipelines. And there exists a lot of liquefied natural gas technology using natural gas pipeline network pressure energy, such as the patent US6023942 [13], US6209350B1 [14], US6449982B1 [15], US6378330B1 [16]. Those processes are all single-utilization technology. Wendong $\mathrm{Xu}$ [17], researched the electricity-ice integrated process using natural gas pipeline network pressure energy. Hui Zhang [18] designed integrated technology research on pressure energy of natural gas pipe network and do the EUD analysis, whose energy efficiency is a little lower. 
Existing electricity-CNG single-compression process using natural gas pressure energy has the characteristics of large expansion ratio, big compression ratio, strict equipment conditions demanding and low energy efficiency. In order to solve the disadvantages above, electricity-CNG multi-compression process using natural gas pressure energy is designed by author in this paper.

\section{Process design}

The components of natural gas are shown in Table 1 . The pressure and temperature of inlet natural gas are $4.0 \mathrm{MPa}$ and $20^{\circ} \mathrm{C}$, respectively. The input of the process is $13800 \mathrm{Nm} 3 / \mathrm{h}$, part of natural gas is compressed to $25 \mathrm{MPa}$ and enters the $\mathrm{CNG}$ storage tank; the other part is input into the urban natural gas pipeline network with the pressure of $0.4 \mathrm{MPa}$.

Table 1 Components of the natural gas

\begin{tabular}{cccccccccc}
\hline Components & $\mathrm{CH}_{4}$ & $\mathrm{C}_{2} \mathrm{H}_{6}$ & $\mathrm{C}_{3} \mathrm{H}_{8}$ & $i-\mathrm{C}_{4} \mathrm{H}_{10}$ & $n-\mathrm{C}_{4} \mathrm{H}_{10}$ & $\mathrm{~N}_{2}$ & $\mathrm{CO}_{2}$ & $\mathrm{He}$ & Total \\
\hline Mole fraction/\% & 94.70 & 0.55 & 0.08 & 0.01 & 0.01 & 1.92 & 2.71 & 0.02 & 100 \\
\hline
\end{tabular}

\section{Single-compression process design}

In the paper, electricity-CNG single-compression process using natural gas pressure energy acts as the compared process by multi-compression process.

The flow sheet of electricity-CNG single-compression process using natural gas pressure energy is shown in Fig.1. After filtration and measurement, upstream gas is divided into two parts, one part of the gas expands to $0.4 \mathrm{MPa}$ in the EXP 03, driving the generator to generate electricity, then low-temperature gas after expansion enters HX 04 and then goes into downstream medium-pressure network; the other part of natural gas is compressed to $25 \mathrm{MPa}$ in the COMP 02. High-temperature CNG exchanges heat with cryogenic natural gas in the HX 06, and then CNG enters the CNG cylinders, CNG tanks and CNG stations.

Electricity produced in the process can be only used in self-control, remote transmission etc..

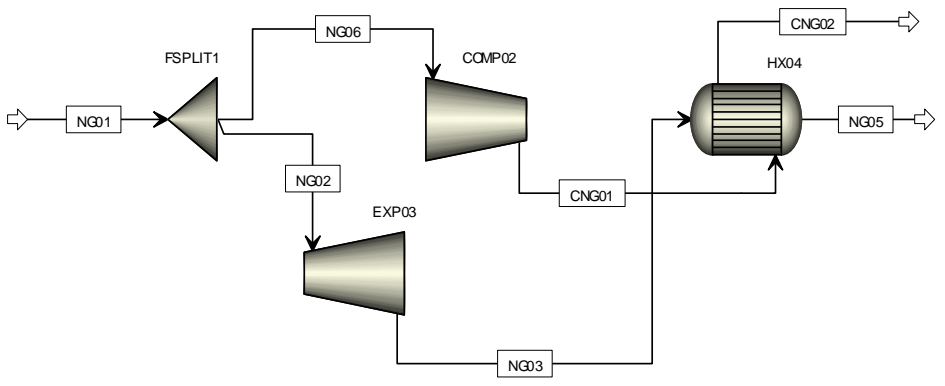

Fig.1 The flow sheet of electricity-CNG single-compression process using natural gas pressure energy

\section{Multi-compression process design}

The flow sheet of electricity-CNG multi-compression process using natural gas pressure energy is shown in fig.2. Upstream gas can be divided into two parts, one part expands to 1.6 MPa, then carries on the secondary expansion to $0.4 \mathrm{MPa}$ and last enters the downstream medium pressure network; the other part is compressed to $16 \mathrm{MPa}$, then goes on the secondary compression to $25 \mathrm{MPa}$ and last enters the CNG storage tank. The raw material gas parameters of multi-compression process are shown in table 1.

In the process, after filtration and measurement, upstream gas is divided into two parts, one part of the gas expands for one-level to 1.6 MPa in the EXP 04, driving the generator to generate electricity, then through the EXP 05 natural gas goes on the secondary expansion to $0.4 \mathrm{MPa}$, driving generator. Low-temperature gas after expansion enters HX 06 and then goes into downstream medium-pressure network; the other part of natural gas is compressed to $16 \mathrm{MPa}$ in the COMP 02 for the one-level 
compression, and then is compressed to $25 \mathrm{MPa}$ for the two-level compression in the COMP 03. CNG at high-temperature exchanges heat with cryogenic natural gas in HX06, and then CNG enters the CNG cylinders, CNG tanks and CNG stations. Not only the electricity produced in the process be can used in self-control, remote transmission and CNG compressor in part, and but can be also used in the surrounding offices and so on.

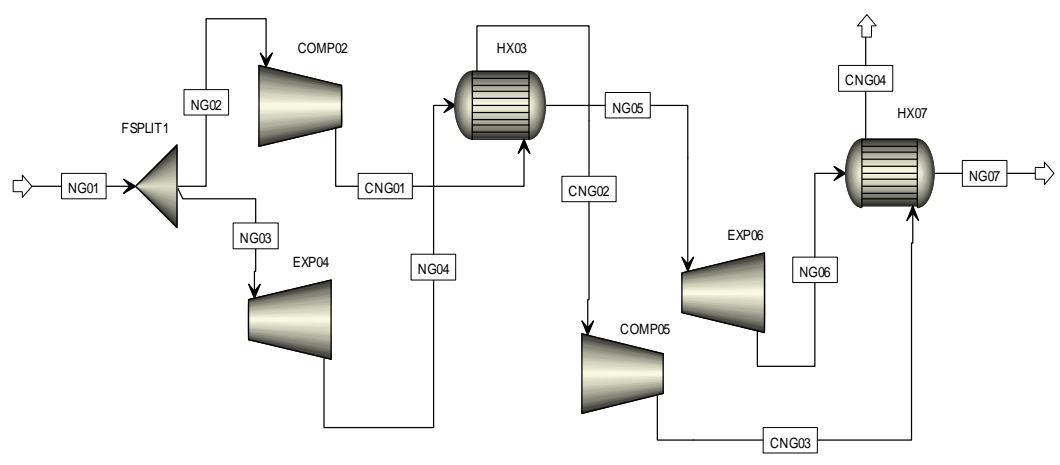

Fig.2 The flow sheet of electricity-CNG multi-compression process using natural gas pressure energy

\section{Process simulation}

Flow sheet simulations are implemented with the commercial Aspen Plus software. To simplify the simulations, it is assumed that the systems are steady state flowing. As most of the inputs involved in two processes are hydrocarbons, SRK (Redilich Kwong Soave) equation is chosen as the model for the thermodynamic properties and phase equilibrium computation in this paper.

In the simulations, the expansion and compression process is deemed as isentropic process and the specific parameters are listed in the table 2 and table 3, respectively. Heat loss in the heat exchanger is ignored and the specific pressure drop is $0 \mathrm{MPa}$.

Table 2 The parameters of natural gas in the single-compression process

\begin{tabular}{ll}
\hline \multicolumn{1}{c}{ Item } & \multicolumn{1}{c}{ Value } \\
\hline The input pressure of one-level expander & $4.0 \mathrm{MPa}$ \\
The output pressure of one-level expander & $0.4 \mathrm{MPa}$ \\
The input pressure of one-level compressor & $4.0 \mathrm{MPa}$ \\
The output pressure of one-level compressor & $25 \mathrm{MPa}$ \\
Isentropic efficiency of one-level expander & 0.8 \\
Mechanical efficiency of one-level expander & 0.9 \\
Isentropic efficiency of one-level compressor & 0.75 \\
Mechanical efficiency of one-level compressor & 0.9 \\
\hline Table 3 The parameters of natural gas in the multi-compression process \\
\hline \multicolumn{1}{c}{ Item } & $\mathrm{Value}$ \\
\hline The input pressure of one-level expander & $4.0 \mathrm{MPa}$ \\
The output pressure of one-level expander & $1.6 \mathrm{MPa}$ \\
The output pressure of two-level expander & $0.4 \mathrm{MPa}$ \\
The input pressure of one-level compressor & $4.0 \mathrm{MPa}$ \\
The output pressure of one-level compressor & $16 \mathrm{MPa}$ \\
The output pressure of two-level compressor & $25 \mathrm{MPa}$ \\
Isentropic efficiency of one-level expander & 0.8 \\
Mechanical efficiency of one-level expander & 0.9 \\
Isentropic efficiency of two-level expander & 0.8 \\
\hline
\end{tabular}




\begin{tabular}{ll}
\hline Mechanical efficiency of two-level expander & 0.9 \\
Isentropic efficiency of one-level compressor & 0.75 \\
Mechanical efficiency of one-level compressor & 0.9 \\
Isentropic efficiency of two-level compressor & 0.75 \\
Mechanical efficiency of two-level compressor & 0.9 \\
\hline
\end{tabular}

Table 4 Compositions and exergy values of key streams in the single-compressing process

\begin{tabular}{ccccccc}
\hline Stream & $\begin{array}{c}\text { Flow } \\
\text { rate } \times 10^{-3} / \mathrm{kg}^{\prime} \cdot \mathrm{h}^{-1}\end{array}$ & $\mathrm{~T} /{ }^{\circ} \mathrm{C}$ & $\mathrm{p} / \mathrm{MPa}$ & Enthalpy $/ \mathrm{kJ}^{\prime} \mathrm{kg}^{-1}$ & $\begin{array}{c}\text { Entrophy/ } \\
\mathrm{kJ} \cdot \mathrm{kg}^{-1} \cdot \mathrm{K}^{-1}\end{array}$ & $\begin{array}{c}\text { Exergy } \times 10^{-4} / \\
\mathrm{kJ} \cdot \mathrm{h}^{-1}\end{array}$ \\
\hline NG01 & 10 & 20 & 4 & -4234 & -6.9 & -2190.8 \\
NG02 & 6.9 & 20 & 4 & -4234 & -6.9 & -1511.6 \\
NG03 & 6.9 & -74 & 0.4 & -4410 & -6.6 & -1679.8 \\
NG04 & 6.9 & 28 & 0.4 & -4173 & -5.6 & -1718.0 \\
NG05 & 3.1 & 20 & 4 & -4234 & -6.9 & -691.1 \\
CNG01 & 3.1 & 194 & 25 & -3857 & -6.7 & -581.4 \\
CNG02 & 3.1 & 30 & 25 & -4385 & -8.0 & -615.9 \\
\hline
\end{tabular}

Table 5 The electricity supply and demand of key equipment in the single-compression process

\begin{tabular}{cc}
\hline Equipment & Power $/ \mathrm{kW}$ \\
\hline COMP02 & -325 \\
EXP03 & 337 \\
\hline
\end{tabular}

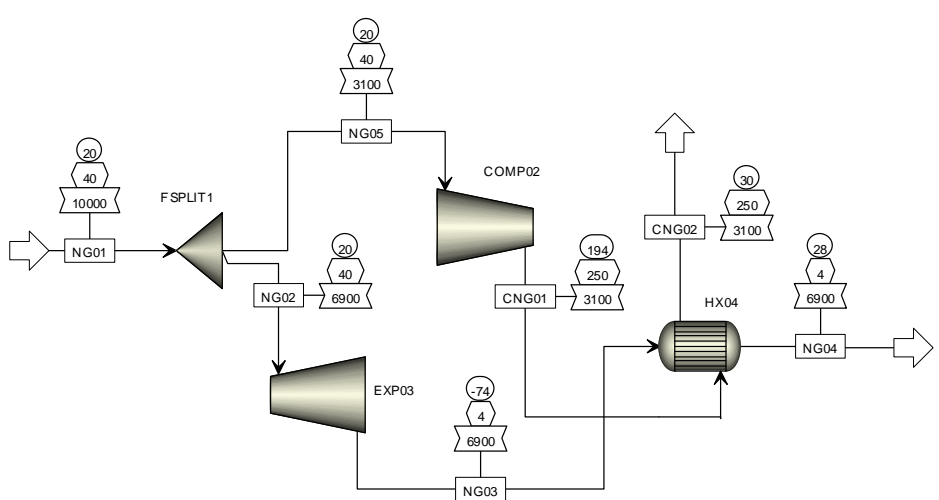

Fig. 3 The flow sheet of process simulation of electricity-CNG single-compression process using pressure energy

Table 6 Compositions and exergy values of key streams in the multi-compression process

\begin{tabular}{ccccccc}
\hline Stream & $\begin{array}{c}\text { Flow } \\
\text { rate } \times 10^{-3} / \mathrm{kg} \cdot \mathrm{h}^{-1}\end{array}$ & $\mathrm{~T} /{ }^{\circ} \mathrm{C}$ & $\mathrm{P} / \mathrm{MPa}$ & $\begin{array}{c}\text { Enthalpy } / \mathrm{kJ} \cdot \mathrm{kg}^{-} \\
1\end{array}$ & $\begin{array}{c}\text { Entrophy/ } \\
\mathrm{kJ} \cdot \mathrm{kg}^{-1} \cdot \mathrm{K}^{-1}\end{array}$ & $\begin{array}{c}\text { Exergy } \times 10^{-4} / \\
\mathrm{kJ} \cdot \mathrm{h}^{-1}\end{array}$ \\
\hline NG01 & 10 & 20 & 4 & -4234.2 & -6.85 & -2190.8 \\
NG02 & 3.1 & 20 & 4 & -4234.2 & -6.85 & -679.1 \\
NG03 & 6.9 & 20 & 4 & -4234.2 & -6.85 & -1511.6 \\
NG04 & 6.9 & -39 & 1.3 & -4329.0 & -6.75 & -1598.1 \\
NG05 & 6.9 & 11 & 1.3 & -4219.8 & -6.33 & -1609.9 \\
NG06 & 6.9 & -49 & 0.4 & -4322.3 & -6.21 & -1704.4 \\
NG07 & 6.9 & 5 & 0.4 & -4220.5 & -5.81 & -1717.0 \\
CNG01 & 3.1 & 104 & 10 & -4070.0 & -6.74 & -638.5 \\
CNG02 & 3.1 & 20 & 10 & -4313.1 & -7.47 & -646.2 \\
CNG03 & 3.1 & 98 & 25 & -4158.4 & -7.37 & -608.1 \\
CNG04 & 3.1 & 30 & 25 & -4384.9 & -8.04 & -615.9 \\
\hline
\end{tabular}

Table 7 The electricity supply and demand of key equipment in the multi-compression process

\begin{tabular}{cc}
\hline Equipment & Power/kW \\
\hline COMP02 & -141 \\
EXP04 & 182 \\
COMP05 & -133 \\
EXP06 & 196 \\
\hline
\end{tabular}




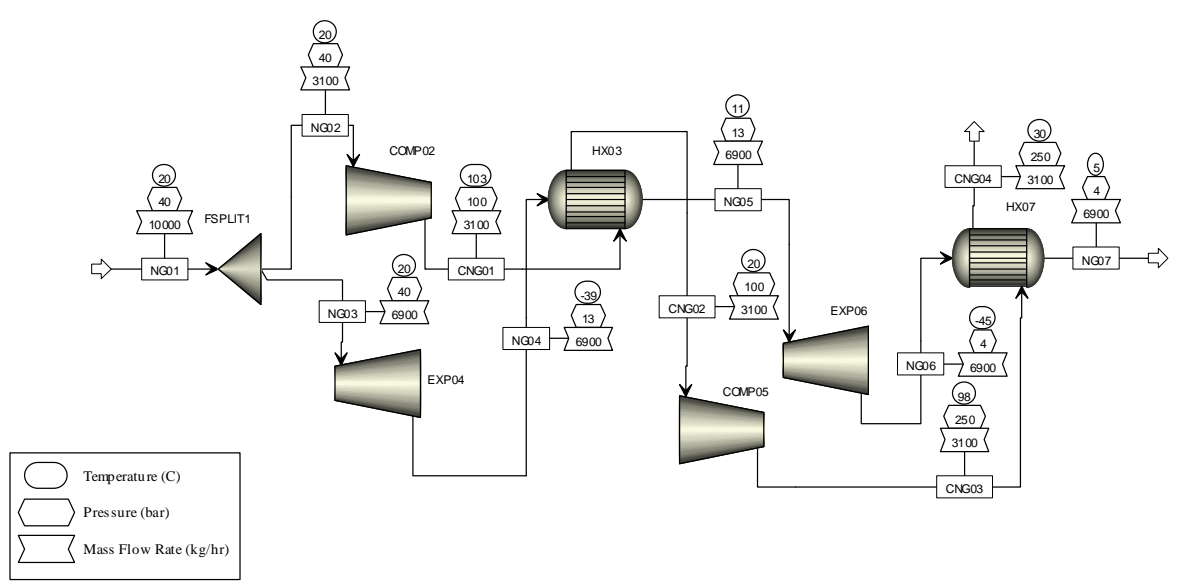

Fig.4 The flow sheet of process simulation of electricity-CNG multi-compression process using pressure energy

Through the above comparison of single-compression and multi-compression process, with the same CNG production, for the single-compression process, the power $337 \mathrm{~kW}$ was produced, the power $325 \mathrm{~kW}$ was consumed by compressor, and the net power of whole system was $12 \mathrm{~kW}$; While for the multi-compression process, the power $378 \mathrm{~kW}$ was produced, the power $378 \mathrm{~kW}$ was consumed by compressors, and the net power of whole system was $104 \mathrm{~kW}$. The former can be only considered for data acquisition, data remote transmission, remote monitoring, explosion-lights, explosion-fan etc., while the latter in addition to the application above, can also supply the surrounding offices.

The temperature difference between the cold stream and the hot stream in the heat exchanger 3 is showed in Fig. 5, in which the minimum temperature difference is $9.1^{\circ} \mathrm{C}$, and the temperature of the cold and hot streams are $10.9^{\circ} \mathrm{C}$ and $20^{\circ} \mathrm{C}$, respectively. The temperature difference between the cold stream and the hot stream in the heat exchanger 7 is showed in Fig. 6, in which the minimum temperature differences of heat exchanger 7 is $24.9{ }^{\circ} \mathrm{C}$, and the temperature of the cold and hot streams are $5.1{ }^{\circ} \mathrm{C}$ and $30^{\circ} \mathrm{C}$, respectively.

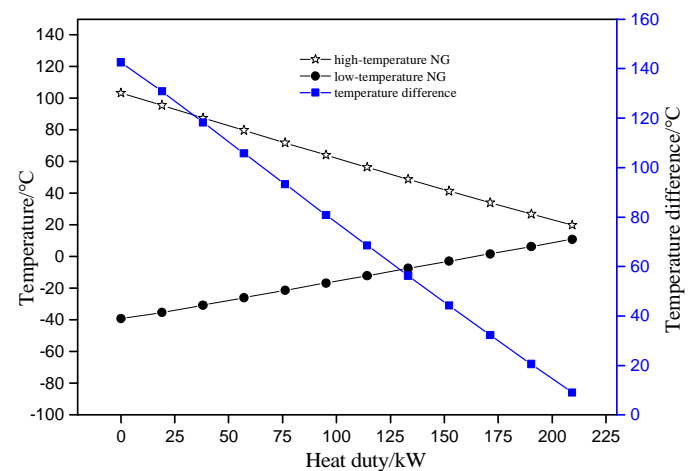

Fig. 5 The temperature difference between the cold stream and the hot stream in heat exchanger HX 03

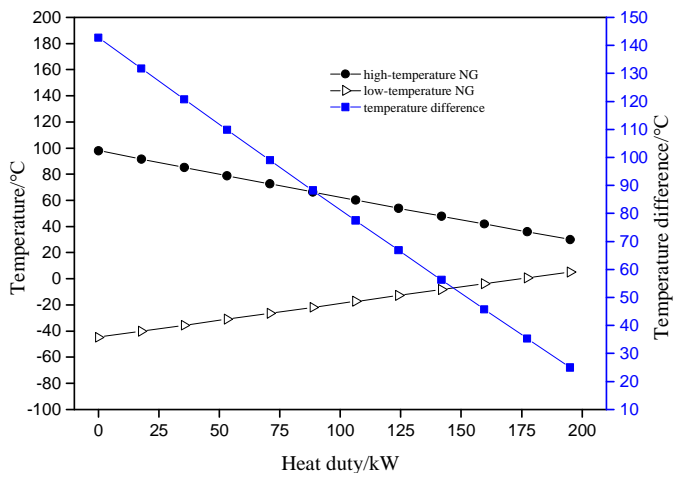

Fig. 6 The temperature difference between the cold stream and the hot stream in heat exchanger HX 07

\section{Thermodynamic analysis}

\section{Exergy analysis}

The exergy [18] is defined by Kotas as maximum work available, accounting for the irreversibility of a process and providing a more detailed tracking mechanism for energy usage. Exergy can be seen as being composed of physical exergy and chemical exergy:

$$
E_{\mathrm{X}}=E_{\mathrm{X}, \mathrm{phs}}+E_{\mathrm{X}, \mathrm{chem}}
$$


Where $E_{\mathrm{X}}$ is the total exergy, $E_{\mathrm{X}, \mathrm{phs}}$ the physical exergy, and $E_{\mathrm{X}, \text { chem }}$ the chemical exergy, respectively.

In a steady state flowing and open system, it is assumed that potential exergy and kinetic exergy are ignored, then the exergy of the stream is calculated by

$$
E_{\mathrm{X}}=\Delta H-T_{0} \Delta S=H-H_{0}-T_{0} \quad\left(S-S_{0}\right)
$$

Where $\Delta H$ is enthalpy change, $\Delta S$ is entropy change, $T_{0}$ is the reference temperature $298.15 \mathrm{~K}, H_{0}$ and $S_{0}$ are enthalpy and entropy at the reference temperature, respectively.

The exergy values of single-compression process are shown in table 4 , and the ones of multi-compression process are shown in table 6. Black box model is used to assess the exergy of single equipment and the whole system. The input total exergy of one system is equal to the sum of output total exergy of the system:

$$
E_{\mathrm{X}, \text { in }}-E_{\mathrm{X}, \text { out }}-E_{\mathrm{X}, \mathrm{loss}}=0
$$

Where $E_{\mathrm{X} \text {,in }}$ is the total input exergy; the total output exergy includes two forms of exergy, namely, the effective exergy $E_{\mathrm{X} \text {,out }}$ and the exergy loss $E_{\mathrm{X}, \text { loss. }}$. The exergy efficiency is calculated as a ratio of the effective exergy $E_{\mathrm{X} \text {,out }}$, to the total input exergy $E_{\mathrm{X} \text {,in }}$.

$$
\eta=\frac{E_{X, o u t}}{E_{X, \text { in }}}
$$

Correspondingly, the exergy loss rate is calculated as

$$
\xi=1-\eta=\frac{E_{X, \text { loss }}}{E_{X, \text { in }}}
$$

Exergy efficiencies of key equipment of the single-compression process are shown in Table 8, and exergy efficiencies of key equipment of the multi-compression process are shown in Table 9.

Table 8. Exergy efficiency of units in the single-compression process

\begin{tabular}{cc}
\hline Unit & Efficiency of exergy $(\eta) \%$ \\
\hline COMP02 & 93.8 \\
EXP03 & 72.1 \\
HX04 & 90.3 \\
The whole process & 38.5 \\
\hline
\end{tabular}

Table 9. Exergy efficiency of units in the multi-compression process

\begin{tabular}{cc}
\hline Unit & Efficiency of exergy $(\eta) \%$ \\
\hline COMP02 & 80.0 \\
HX03 & 65.3 \\
EXP04 & 75.7 \\
COMP05 & 75.1 \\
EXP06 & 74.7 \\
HX07 & 61.9 \\
The whole process & 57.9 \\
\hline
\end{tabular}


As shown in the table 8, the exergy efficiency of COMP 02, EXP 03 and HX 04 are 93.8\%, 72.1\% and $90.3 \%$ respectively, and exergy efficiency of the whole single-compression process is $38.5 \%$. As shown in the table 9, the exergy efficiency of COMP 02, HX 03, EXP 04, COMP 05, EXP 06 and HX 07 are $80 \%, 65.3 \%, 75.7 \%, 75.1 \%, 74.7 \%, 61.9 \%$ respectively, and exergy efficiency of the whole multi-compression process is $57.9 \%$.

Compared to single-compression process, each equipment exergy utilization rate in multi-compression process is not so high, but the exergy efficiency of the whole system is higher than single-compression process, and the exergy efficiency is increased by $50.4 \%$.

\section{Energy utilization diagram (EUD) analysis}

The conventional exergy analysis is based on the exergy balance calculation. The EUD analysis $[19,20]$ is a better approach to analyze the process energy efficiency, proposed by Ishida in 1982, and revealing fundamental mechanism of the exergy loss in the operation units. EUD analysis could analyze the weak point of the process and provide a way to improve the performance of the process.

In the EUD, the energy process is made up of an energy donor and an energy acceptor. The energy level $A$, a new intensive parameter, is introduced in the EUD, a ratio of the exergy change $\Delta E$ to the enthalpy change $\Delta H$ in the process:

$$
A=\Delta E / \Delta H
$$

The energy level exists difference between the energy donor and the energy acceptor drives the process. The area between the energy donor curve and the energy acceptor curve in the EUD represents the exergy loss of the process.

In a steady state system, the exergy change can be calculated with

$$
\begin{aligned}
& \Delta E=\Delta H-T_{0} \Delta S \\
& A=1-T_{0} \Delta S / \Delta H
\end{aligned}
$$

In the heat exchanger subsystem, $\Delta H$ is the heat duty $Q$, and $\Delta S$ is $Q / T$, Eq. is equal to

$$
A=\Delta E / \Delta H=1-T_{0} / \mathrm{T}
$$

In the power subsystem, $\Delta H$ is equal to $W$ and $\Delta S=0$, Eq. becomes

$$
A=\Delta E / \Delta H=1-T_{0} \Delta S / \Delta H=1
$$

This means that the energy level of the energy donor in the compressor and the energy acceptor in the expansion are always equal to one; its energy level of the energy acceptor can be calculated. And $\Delta H, \Delta S$ can be calculated by below equations.

$$
\begin{aligned}
& \Delta H=n C_{p}\left(T_{\text {out }}-T_{\text {in }}\right) \\
& \Delta S=n \ln \left[\left(\frac{T_{\text {out }}}{T_{\text {in }}}\right)^{C_{p}}\left(\frac{P_{\text {out }}}{P_{\text {in }}}\right)^{-R}\right]
\end{aligned}
$$

EUD exergy loss analysis method can reflect the key internal reason in the process, factually showing the exergy loss in the energy transfer process. The energy level difference is the driving force of energy transfer process between energy donor and energy acceptor. The point of the smallest driving force is the optimization and improvement point.

The EUD of the multi-compression process is shown in Fig.7, in which the energy level is $Y$-axis, while the corresponding heat duty is $X$-axis. Exergy loss of each unit can be calculated from the area confined within each pair of curves, the results are shown in Table 10. 


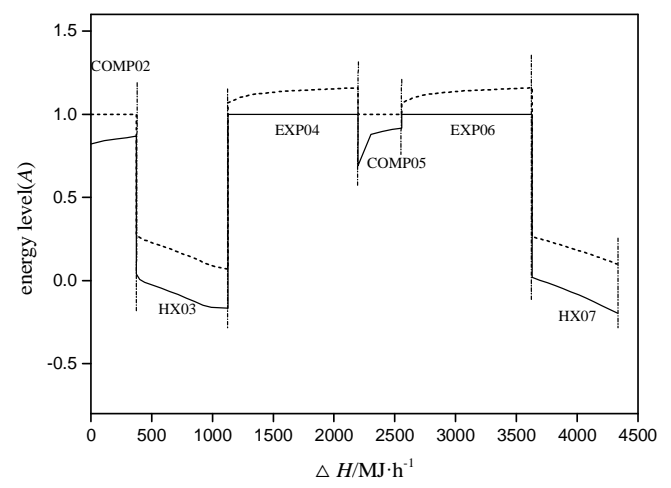

Fig.7 EUD of the multi-compression process COMP-compressor; EXP-expansion; HX-heat exchanger; _-acceptor; donor

Table 10 Exergy loss of the key equipment in the multi-compression process

\begin{tabular}{cccc}
\hline Item & Exergy loss/kW & Curves & Fraction of total exergy/\% \\
\hline Total exergy loss & 214.5 & - & 100 \\
Compressor1 & 15.9 & COMP02 & 7.41 \\
Compressor2 & 13.3 & COMP05 & 6.20 \\
Expansion 1 & 40.1 & EXP04 & 18.69 \\
Expansion 2 & 40.1 & EXP06 & 18.69 \\
Heat exchanger1 & 53.0 & HX03 & 24.72 \\
Heat exchanger2 & 52.1 & HX07 & 24.29 \\
\hline
\end{tabular}

As shown in the table 10, for heat exchanger 1, the exergy loss is due to the large temperature difference between the cool and hot streams. According to Fig.5, the energy level of the energy donor in heat exchanger 1 is 0.27 , which is 0.44 higher than the energy level of corresponding acceptor. Difference between energy levels of the donor and acceptor causes the large exergy loss in the heat exchanger 1.For expansion 1 and 2, the exergy loss is due to the large expansion ratio. The analysis results show that the heat exchangers 1 and 2, the expansion 1 and 2 are the key units, leaving rooms for further improvement. With the increase of the compression or expansion level, the efficiency of energy utilization is on the rise, but the energy utilization growth rate is reduced and the cost of corresponding equipment increases. Therefore 2 or 3 level expansion or compression is the good choice.

\section{Techno-economic analysis}

In the traditional single-compression process, $4276 \mathrm{Nm}^{3} / \mathrm{h}(3.1 \mathrm{t} / \mathrm{h}) \mathrm{CNG}$ products need $274 \mathrm{~kW}$ power. While in the multi-compression process, not only are there the products of $4276 \mathrm{Nm}^{3} / \mathrm{h}(3.1 \mathrm{t} / \mathrm{h})$ CNG, there also exists the extra power of $104 \mathrm{~kW}$. The main investments are the equipment, which are shown in the Table 11.

Table 11 Investments of multi-compression process

\begin{tabular}{ccc}
\hline Number & Item & Total Price/million CNY \\
\hline 1 & Expander unis & 4.80 \\
2 & Compressor units & 2.70 \\
3 & Heat exchangers & 1.60 \\
4 & Other ancillary equipment & 1.20 \\
5 & Civil engineering and covering & 0.90 \\
6 & Total & 11.2 \\
\hline
\end{tabular}

Total investments of the multi-compression process, in the case of 103.6 thousand $\mathrm{Nm}^{3} / \mathrm{d} \mathrm{CNG}$ capacity, are 11.2 million CNY. And the electricity saving benefits are $3.3 \times 10^{6} \mathrm{kWh}$ per year. Counting industrial electricity price by $1.5 \mathrm{CNY} / \mathrm{kWh}$, annual electricity saving benefits are 4.97 million CNY. The payback period is 2.25 years. 


\section{Conclusion}

Through the comparison of single-compression process and multi-compression process, with the same CNG production, for the single-compression process, the power $337 \mathrm{~kW}$ was produced, the power $325 \mathrm{~kW}$ was consumed by compressor, and the net power of whole system was $12 \mathrm{~kW}$; While in multi-compression process, the power $378 \mathrm{~kW}$ was produced, the power $274 \mathrm{~kW}$ was consumed by compressors, and the net power of whole system was $104 \mathrm{~kW}$.

The exergy analysis of the proposed process was carried out. In addition, a method of the energy utilization diagram was used to identify the operation units with large exergy loss. The analysis results showed that the exergy efficiency of the multi-compression process is 57.9\%; Compared to the single-compressing process, exergy efficiency of the multi-compression process was improved by $50.4 \%$. The exergy analysis and the EUD analysis had identified the units with low exergy efficiency and the units with large exergy loss, the results could then be used to further optimize the entire process.

Techno-economic analysis of the multi-compression process was carried out. The results showed that total investment of the process, in the case of 103.6 thousand $\mathrm{Nm}^{3} / \mathrm{d} \mathrm{CNG}$ capacity, was 11.2 million CNY. The payback period was 2.25 years.

\section{REFERENCES}

[1] J.J.Chen , S.L.Wang: Shan Xi Energy and Conservation., 4, 14-16(2006).

[2] H. Zhang, X.X. Li , W.D.Xu : Gas \& Heat., 35(7), A35-A37(2015).

[3] X.X.Li,, H.Zhang, W.D.Xu : Advances in Engineering Research., 17, 1155-1160(2015).

[4] C.Howard , P.Oosthuizen , B.Peppley: Applied Thermal Engineering., 31(13), 2165-2170(2011).

[5] S.Sanaye , N.A.Mohammadi: Energy., 40(1), 358-369(2012).

[6] C.M.An: South China University of China., (2013).

[7] Z.Zheng, S.L.Wang, X.R.Xu : Gas \& Heat., (12), 33-36(2009).

[8] D.M.Shen, Fernandes F., Simqes-Moreira J.R.: Hydrocarbon Processing., 85(1), 47-50(2006).

[9] J.Wen , M.F.Xu : Natural Gas Industry., (07), 106-108(2007).

[10] S.B.Liang, Z.Q.Fang , C.Zhu: Refrigeration., (01), 20-22(1996).

[11] S.B.Liang, Z.Q.Fang, C.Zhu: Journal of Refrigeration., (03), 27-29(1995).

[12] Y.Q.Xiong, B.Hua, D.X.Luo: Modern Chemical Industry., (01), 49-52(2007).

[13] R.T.Eugene, R.B.Ronald, T.C.Eric: The United States: 6023942, (2004).

[14] E.L Kimble: The United States: 6209350B1, (2001).

[15] F.Bestric: The United States: 6449982B1, (2002).

[16] M.Moses, R. B.Ronald, B.S.John: The United States: 6378330B1, (2002).

[17] Q.X.Chen, W.D.Xu, C.M.An: Gas \& Heat., 32(9), A25-A27(2012).

[18] H.Zhang: South China University of China., (2014).

[19] H.G.Jin, M.Ishida:Energy., 18, 615-625(1993).

[20] T.Srinophakun, S.Laowithayangkul, M.Ishida:Energy Convers Manage., 42, 1437-1456(2001). 